e.g. Haystack, FGAN, or the combined use of FGAN with the $100 \mathrm{~m}$ Effelsberg radio telescope, statistical information on $1 \mathrm{~cm}$ size objects in Low-Earth orbit (LEO) can be gained.

With an uncontrolled growth orbital debris could become a major hazard for all human activities in space. Already now orbital debris is a potential risk in two important regions, namely at low Earth altitude, and in the geostationary ring. An indication of the degrading quality of the space environment are the first confirmed collision of an operational spacecraft (Cerise) with a rocket fragment, avoidance manoeuvres of the US Space Shuttle and of unmanned spacecraft in LEO, and the more than 60 replacements of windows of the US Space Shuttle damaged by impacts of small-size debris and meteoroids. Space debris is also degrading astronomical observations as large scale exposures of the sky may contain satellite trails.

This presentation will provide an overview of the terrestrial space debris environment. Sources for the catalogued objects and small-size space debris will be reviewed. Models for the spatial distribution will be presented. Explosions and collisions as the main debris sources will be addressed. Finally, the long-term evolution and debris reduction measures will be reviewed.

\title{
12. ENVIRONMENTAL DISTURBANCES OF ASTRONOMICAL OBSERVATIONS
}

\section{J. Kovalevsky (CERGA, Observatoire de la Côte d'Azur)}

This presentation is aimed at describing several effects, generally of geophysical origin, which contribute, in addition to sky glow or electromagnetic wave interferences, to degrade astronomical observations from the ground.

The efficiency of an astronomical instrument must be considered together with all the atmospheric layers crossed by the incoming light. This optically active element affects the shape of the image and the apparent direction of the observed celestial body. Astrometric measurements may be significantly biased by un-modelled spurious refraction effects. But the most important source of disturbances is the local atmospheric turbulence which is the major factor of astronomical seeing. The height of the boundary layer is function of the existence of heat sources. In day-time, it is governed by the Sun, but in night-time, the presence of buildings, roads, vegetation of various types, has a definite influence on the quality of the atmospheric images, increasing their unstability and introducing sometimes an inclination of the atmospheric layers, causing abnormal refraction.

The chemical composition of the atmosphere has evidently a direct effect on spectroscopy. This is particularly important in millimeter and submillimeter band astronomy, in the research of various interstellar or circumstellar molecules. The atmospheric emissions and opacity in this wavelength domain are major perturbations in recognizing the spectral lines of these extraterrestrial cmissions. Increase in opacity may also occur after major geophysical events such as aerosols or dust from volcanos.

Optical interferometres are very sensitive to any perturbation of the optical path lengths. This can be produced by deformations of the wavefront due to turbulence, and particularly long period deformations in the higher atmospheric layers. Another cause is the variation of the baseline, consequence of seismic activity. Very significant perturbations are produced by oceanic waves which and are important even several hundreds of kilometers away. Storms on the ocean may be sources of important deterioration of interferometric observations.

Some of the atmospheric dynamical effects may be compensated using active or arlaptative optics. It has however to be noted that these techniques correct the wavefront only within a few arc seconds. This is certainly the solution when a single star is being observed, but does not apply for larger field studies. It is also conceivable that is the future, interferometric baselines could be corrected in real time from seismic simultaneous readings. Millimetric and submillimetric observations can get rid of a large part of spectroscopic pollution in hight altitude sites. But most of the ground based astronomical observations will continue to be lighly sensitive to these environmental perturbations.

\section{THE AVOIDANCE OF MAN-MADE POLLUTION IN INTERPLANETARY SPACE}

C. S. L. Keay (University of Newcastle)

At the 20th General Assembly of the International Astronomical Union in Baltimore, U.S.A., in 1988 members of Commission 22 and several Commissions in what is now the Division of Plan- 